\title{
Evaluation of the Facial Recess and Cochlea on the Temporal Bone of Stillbirths regarding the Percutaneous Cochlear Implant
}

\author{
Gabriela Pereira Bom Braga ${ }^{1}$ Eloisa Gebrim ${ }^{2}$ Ramya Balachandran ${ }^{3} \quad$ Jack Noble $^{4}$ Robert Labadie ${ }^{3}$ \\ Ricardo Ferreira Bento ${ }^{1}$ \\ ${ }^{1}$ Department of Otolaryngology, Faculdade de Medicina, \\ Universidade de São Paulo, São Paulo, SP, Brazil \\ ${ }^{2}$ Department of Radiology, Faculdade de Medicina, Universidade de \\ São Paulo, São Paulo, SP, Brazil \\ ${ }^{3}$ Department of Otolaryngology Head and Neck Surgery, Vanderbilt \\ University, Nashville, Tennessee, United States \\ ${ }^{4}$ Department of Otolaryngology, Vanderbilt University, Nashville, \\ Tennessee, United States \\ Int Arch Otorhinolaryngol 2018;22:260-265.

\begin{abstract}
Address for correspondence Gabriela Pereira Bom Braga, MD, PhD, Departmento de Otolaringologia, Faculdade de Medicina, Universidade de São Paulo, Av. Dr. Eneas de Carvalho Aguiar, 255, $6^{\circ}$ andar, sala 6167, São Paulo, SP, 01246-903, Brazil (e-mail: gabrielabombraga36@gmail.com).
\end{abstract}

\begin{abstract}
Keywords

- cochlear implantation

- middle ear

- hearing loss, sensorineural

- temporal bone / anatomy

- cochlea

- experimental studies
\end{abstract}

Introduction The literature shows that there are anatomical changes on the temporal bone anatomy during the first four years of life in children. Therefore, we decided to evaluate the temporal bone anatomy regarding the cochlear implant surgery in stillbirths between 32 and 40 weeks of gestational age using computed tomography to simulate the trajectory of the drill to the scala timpani avoiding vital structures.

Objectives To measure the distances of the simulated trajectory to the facial recess, cochlea, ossicular chain and tympanic membrane, while performing the minimally invasive cochlear implant technique, using the Improvise imaging software (Vanderbilt University, Nashville, TN, US).

Methods An experimental study with 9 stillbirth specimens, with gestational ages ranging between 32 and 40 weeks, undergoing tomographic evaluation with individualization and reconstruction of the labyrinth, facial nerve, ossicular chain, tympanic membrane and cochlea followed by drill path definition to the scala tympani. Improvise was used for the computed tomography (CT) evaluation and for the reconstruction of the structures and trajectory of the drill.

Results Range of the distance of the trajectory to the facial nerve: 0.58 to $1.71 \mathrm{~mm}$. to the ossicular chain: 0.38 to $1.49 \mathrm{~mm}$; to the tympanic membrane: 0.85 to $1.96 \mathrm{~mm}$; total range of the distance of the trajectory: 5.92 to $12.65 \mathrm{~mm}$.

Conclusion The measurements of the relationship between the drill and the anatomical structures of the middle ear and the simulation of the trajectory showed that the middle ear cavity at 32 weeks was big enough for surgical procedures such as cochlear implants. Although cochlear implantation at birth is not an indication yet, this study shows that the technique may be an option in the future. received

June 10, 2017

accepted

August 4, 2017

published online

October 25, 2017
Copyright (e 2018 by Thieme Revinter

Publicações Ltda, Rio de Janeiro, Brazil
License terms

10.1055/s-0037-1606612. ISSN 1809-9777.

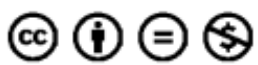




\section{Introduction}

In recent decades, technological advances such as cochlear implants enabled patients with hearing loss to regain the ability to hear. According to recent incidence studies, 14.5\% of the population has some form of disability, and out of this number, $5.2 \%$ declare inability to hear, with $3.9 \%$ of moderate hearing loss, $0.9 \%$ of severe hearing loss, and $0.4 \%$ of profound hearing loss. ${ }^{1}$ The ear derives from the ectoderm of the cephalic part of the embryo, originating from one round vesicle. The otocyst cavity contains the endolymph and the organ of Corti. The membranous labyrinth reaches its maturity and remains with the dimensions acquired on the fifth month of intrauterine life. ${ }^{2-4}$ The inner ear appears on the ninth week as the endolymphatic duct system including mesenchymal tissue into which an early vacuolated perilymphatic forms the labyrinth system. ${ }^{2}$ The cartilaginous otic capsule forms the labyrinth. On the twenty-third week, the cochlea reaches its full size, and the periotic spaces are well-formed., ${ }^{2,5}$ The postnatal growth of the temporal bone is much discussed. For a long time, it was believed that the morphology and spatial orientation of the labyrinth did not change significantly after birth. ${ }^{6}$ However, it appears that the cranium presents a bimodal growth curve between 1 and 4 years of age and again during puberty, which can have a significant impact on the anatomy of the temporal bone. ${ }^{7,8}$ It has been suggested that these changes take place in the tympanic and mastoid processes and in the squamous portion of the temporal bone. ${ }^{9}$

Regarding the relevant anatomy to the cochlear implant, there are significant differences between children and adults. $^{10,11}$ Lloyd, in $2010,{ }^{7}$ presented evidence that the basal turn of the cochlea can change its orientation relative to the facial recess during growth, which can influence the angle of insertion of the electrode. ${ }^{10,12}$

As Moberly et al described in 2015, early stimulation of the cerebral cortex yields better results on hearing rehabilitation. ${ }^{13}$ Replacing the function of the organ of Corti and electrically stimulating the ganglion cells and nerve endings of the auditory nerve, cochlear implants are used in the hearing rehabilitation of patients with severe to profound hearing loss.

Despite the safety of the procedure having increased due to technological advances, there are still complications, especially in younger patients, in whom the noble anatomical structures (the facial nerve, the chorda tympani nerve and the cochlea) have different angles and are in different positions compared with adults. ${ }^{14-16}$ The current techniques to access the cochlea use visual feedback (with the aid of a microscope) and facial nerve monitoring to avoid these structures. The facial nerve has the most risk of injury because of the nerve recess approach. ${ }^{5}$ In order to increase the safety of the procedure, reduce the surgical and healing times, and accelerate the activation of the cochlear implants, minimally invasive approaches using robotic techniques have been proposed. This surgical technique has been approved by the FDA to be used on adults and children. ${ }^{17-23}$

The procedure is based on the fact that it is possible to pass a drill through the temporal bone after a previous tomo- graphic planning, with the anatomical study of the noble structures of the mastoid through the trajectory using image-guided techniques, while critical structures of the temporal bone are avoided. ${ }^{17-19}$

\section{Objectives}

The objective of the present study was to evaluate the simulated trajectory of the drill on the computed tomography (CT) scan used in the percutaneous cochlear implant technique and its viability in neonates through:

1. A measurement of the simulated trajectory of the drill between the cortical temporal bone and the cochleostomy, calculating the mean, median and standard deviation.

2. An assessment of the mean, median and standard deviation of the distances between the simulated trajectory to the ossicular chain, tympanic membrane and facial nerve.

\section{Methods}

\section{Study Design}

This was an experimental study.

This study was approved by the Ethical Committee of one of our institutions (under CAAE number 51243215.2.0000.065).

\section{Inclusion Criteria}

The corpses that did not show anatomical malformations in the middle and inner ear structures or in bone density during the tomographic evaluation were included in the study.

Methodology

A tomographic study was performed using the machine Discovery CT 750 HD (General Electric Healthcare, Little Chalfont, UK) (CT Protocol- volumetric acquisition helical Hi-res rotation 0.8 seconds with collimation of $0.625 \mathrm{~mm}$ and $0.312 \mathrm{~mm}$ range KV $140 \mathrm{~mA}: 400 \mathrm{FOV}$ - determined by measuring the laterolateral diameter of the temporal region in digital radiography and reconstruction Ultra High Definition). Held in the Radiology Laboratory of one of our institutions.

The specimens were placed in the scanner in the supine position with the head in hyperextension with the aid of a cushion, forming a straight angle to the base of the scanner. The images were made in the coronal and axial planes with a thickness of $0.6 \mathrm{~mm}$. The images were transferred to the Improvise (Vanderbilt University, Nashville, TN, US) software, followed by segmentation of the structures involved in the cochlear implant (facial nerve, ossicular chain, tympanic membrane, labyrinth and cochlea). After the segmentation, the software then simulates the trajectory of the drill between the cortex of the temporal bone and the scala tympani through a cochleostomy (-Fig. 1).

In order to define the path between the cortex of the temporal bone and the cochleostomy, we used features of the software that allow us to make points on different locations of the image, keeping the symmetry between sides. Thus, the first point was positioned in the scala tympani, and the second one on the cortex of the temporal bone, having 


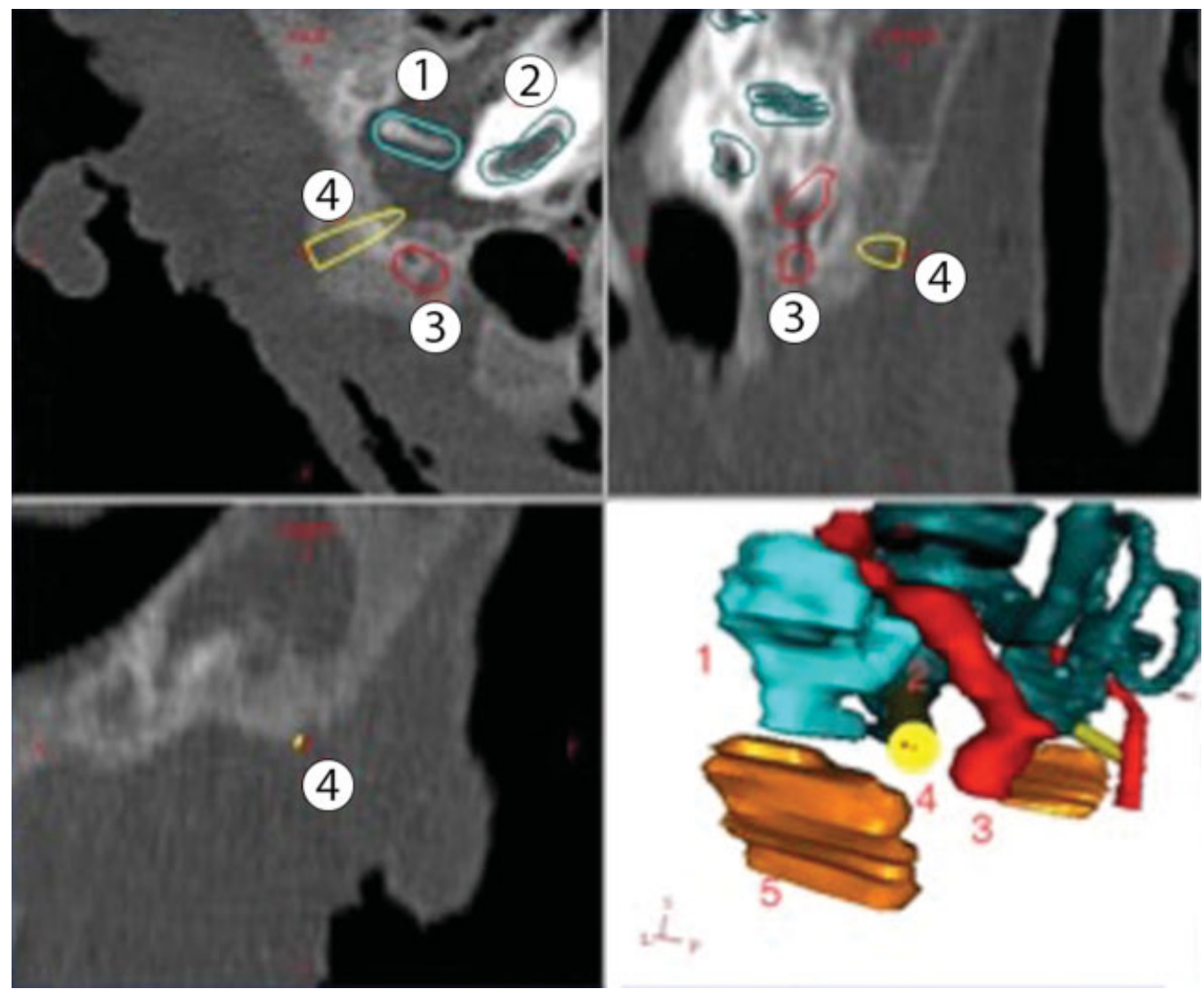

Fig. 1 Four individual plans of reconstructed anatomy in the Improvise software: axial, coronal, sagittal and 3D; 1- ossicular chain; 2- cochlea; 3-facial nerve; 4- trajectory; 5- tympanic membrane.

as limits the facial nerve, the ossicular chain and the tympanic membrane. To simulate the thickness of the drill, a cylinder (yellow) with a radius of $0.5 \mathrm{~mm}$ was created. The axial plane was chosen to perform the demarcation of the defining points of the trajectory, because it presents a more accurate identification of the scala tympani than other planes.

We then measured the shortest distances between: the facial nerve and the trajectory ( - Fig. 2 ); the ossicular chain and the trajectory (-Fig. $\mathbf{3}$ ); the tympanic membrane and the trajectory ( - Fig. 4 ); and the cortex of the temporal bone and the scala tympani.

\section{Results}

Tomographic images of the mastoid of nine specimens of stillbirths that met the inclusion criteria were evaluated. The data were transferred to an Excel (Microsoft Corporation, Redmond, WA, US) spreadsheet, in which we calculated the mean, median and standard deviation and applied the paired samples Student $t$ test ( $\mathbf{- T a b l e s} \mathbf{1}$ and $\mathbf{2}$ ).

The measurements of the trajectory of the drill to the facial nerve ranged from $0.58 \mathrm{~mm}$ to $1.71 \mathrm{~mm}$; for the trajectory to the ossicular chain, they ranged from $0.38 \mathrm{~mm}$ to $1.49 \mathrm{~mm}$. The tympanic membrane was between $0.85 \mathrm{~mm}$ and $1.96 \mathrm{~mm}$ away from the simulated drill path; and the trajectory from the cortex of the temporal bone to

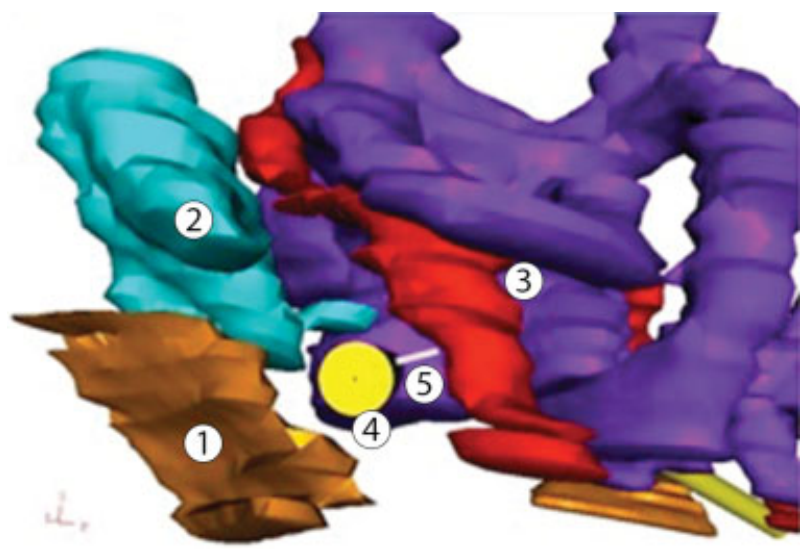

Fig. 2 Shortest distance between the trajectory and the facial nerve: 1- tympanic membrane; 2- ossicular chain; 3-facial nerve; 4- trajectory; 5- distance between the trajectory and the facial nerve.

the scala tympani ranged from $5.92 \mathrm{~mm}$ to $12.65 \mathrm{~mm}$. The paired samples correlations between the right and left sides were evaluated. The first one was the facial nerve; then, the ossicular chain, followed by the tympanic membrane and the trajectory. The related correlation results and significance are respectively described as: facial nerve $-0.598 \mathrm{~mm}$ and $0.089 \mathrm{~mm}$; ossicular chain $-0.252 \mathrm{~mm}$ and $0.514 \mathrm{~mm}$; tympanic membrane $-0.396 \mathrm{~mm}$ and $0.291 \mathrm{~mm}$; and trajectory $-0.958 \mathrm{~mm}$ and $0.0 \mathrm{~mm}$. The paired samples $t$ test 


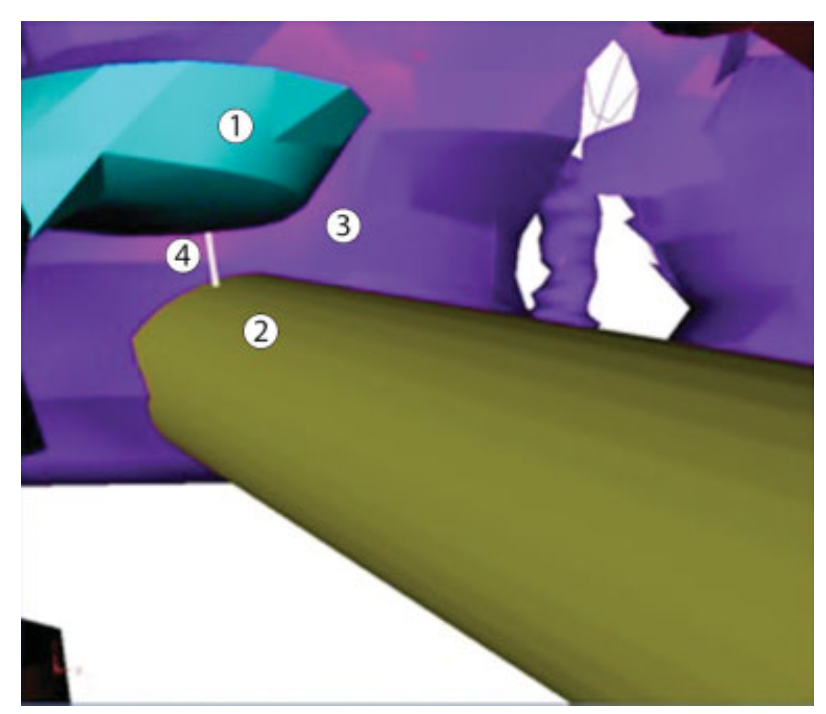

Fig. 3 Shortest distance between the trajectory and the ossicular chain: 1- stapes; 2- trajectory; 3-cochlea; 4- distance between the trajectory and the stapes.

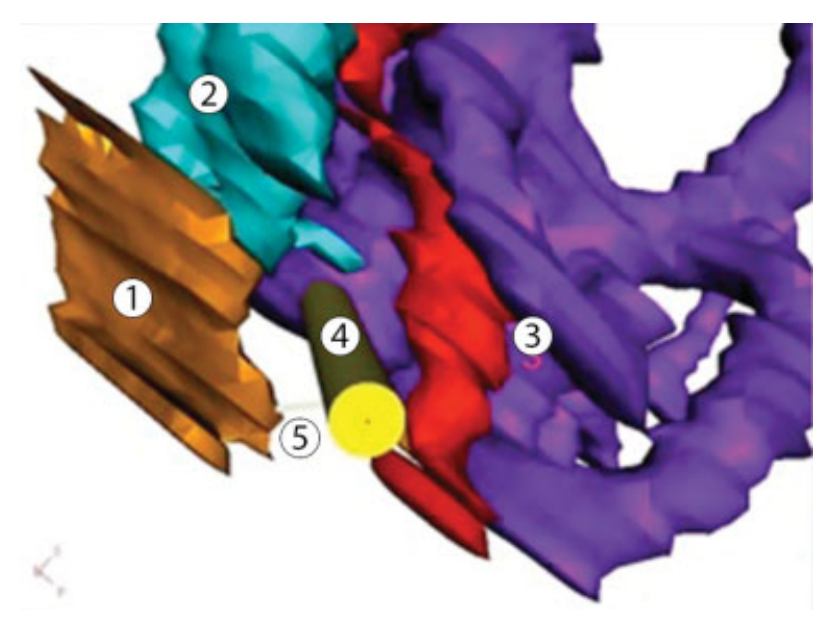

Fig. 4 Shortest distance between the eardrum and the trajectory: 1tympanic membrane; 2- ossicular chain; 3-facial nerve; 4- trajectory; 5- distance between the trajectory and the tympanic membrane.
Table 2 Paired samples statistics

\begin{tabular}{|l|l|l|l|l|l|}
\hline \multicolumn{2}{|c|}{} & Mean & N & $\begin{array}{l}\text { Standard } \\
\text { deviation }\end{array}$ & $\begin{array}{l}\text { Standard error } \\
\text { of the mean }\end{array}$ \\
\hline \multirow{2}{*}{ Pair 1 } & RFN & 1.2033 & 9 & 0.35107 & 0.11702 \\
\cline { 2 - 6 } & LFN & 1.1389 & 9 & 0.26127 & 0.08709 \\
\hline \multirow{2}{*}{ Pair 2 } & ROC & .9278 & 9 & 0.35192 & 0.11731 \\
\cline { 2 - 6 } & LOC & .9856 & 9 & 0.26698 & 0.08899 \\
\hline \multirow{2}{*}{ Pair 3 } & RTM & 1.4100 & 9 & 0.34673 & 0.11558 \\
\cline { 2 - 6 } & LTM & 1.1100 & 9 & 0.33849 & 0.11283 \\
\hline \multirow{2}{*}{ Pair 4 } & RTRA] & 8.8600 & 9 & 2.12525 & 0.70842 \\
\cline { 2 - 6 } & LTRAJ & 8.9922 & 9 & 2.04218 & 0.68073 \\
\hline
\end{tabular}

Abbreviations: LFN, left facial nerve; LOC, left ossicular chain; LTM, left tympanic membrane; LTRAJ, left trajectory; RFN, right facial nerve; ROC, right ossicular chain; RTML, right tympanic membrane; RTraj, right trajectory.

showed a statistical difference between the relative position of the tympanic membrane and the trajectory from the right to the left sides.

\section{Discussion}

By analyzing the images, we noticed that the middle ear cavity at 32 weeks is well-pneumatized, and it could enable surgical procedures such as cochlear implants. In order to confirm that, we propose a simulated study using an imageguided, minimally invasive technique to access the cochlea in pediatric patients undergoing cochlear implant. This approach involves drilling a narrow path from the cortex of the temporal bone to the cochlea without hitting vital anatomical structures such as the facial nerve, ossicular chain and chorda tympani nerve.

The Improvise software was developed at Vanderbilt University, and it is used to analyze, segment, and measure tomographic images of patients undergoing cochlear implant surgery. After the segmentation of the facial nerve,

Table 1 Distance of the drill's trajectory $(\mathrm{mm})$ to the middle ear structures

\begin{tabular}{|l|l|l|l|l|l|l|l|l|}
\hline $\mathbf{N}=\mathbf{1 8}$ & \multicolumn{2}{|l|}{ Facial nerve } & \multicolumn{2}{l|}{ Ossicular chain } & \multicolumn{2}{l|}{ Tympanic membrane } & \multicolumn{2}{l|}{ Trajectory } \\
\hline & Right & Left & Right & Left & Right & Left & Right & Left \\
\hline $\mathbf{1}$ & 0.99 & 1.24 & 0.86 & 1.29 & 1.06 & 0.85 & 5.92 & 6.68 \\
\hline $\mathbf{2}$ & 1.06 & 0.99 & 0.38 & 1.01 & 1.18 & 1.35 & 6.58 & 6.37 \\
\hline 3 & 1.16 & 1.35 & 1.01 & 0.94 & 1.55 & 0.89 & 9.01 & 8.07 \\
\hline $\mathbf{4}$ & 1.55 & 1.02 & 0.95 & 0.78 & 1.64 & 1.23 & 8.28 & 8.89 \\
\hline $\mathbf{5}$ & 1.71 & 1.23 & 0.85 & 0.66 & 1.96 & 1.32 & 8.41 & 8.98 \\
\hline $\mathbf{6}$ & 0.67 & 0.58 & 0.64 & 0.75 & 1.42 & 0.55 & 8 & 7.81 \\
\hline $\mathbf{7}$ & 1.06 & 1.24 & 1.49 & 0.85 & 1.03 & 0.87 & 9.55 & 10.44 \\
\hline $\mathbf{8}$ & 0.99 & 1.11 & 1.42 & 1.47 & 1.79 & 1.64 & 11.34 & 11.34 \\
\hline $\mathbf{9}$ & 1.64 & 1.49 & 0.75 & 1.12 & 1.06 & 1.12 & 12.65 & 12.35 \\
\hline
\end{tabular}


cochlea, labyrinth, tympanic membrane (used as reference because the chorda tympani nerve could not be found on the specimens) and ossicular chain, the images were meshed, and a three-dimensional (3D) view of the middle ear was obtained. From that, the trajectory was determined using Improvise tools. Once we determined a trajectory, keeping the symmetry in the position of the points on both sides, the measurements can be taken using the math operation tool and obtaining the surface distance quantity. Analyzing the tables, we observed that the simulated trajectory of the drill kept a distance of more than $1 \mathrm{~mm}$ from the structures. Even though this distance may seem too close to the facial nerve, for example, some surgical techniques skeletonize the facial recess or even open it to decompress it. Therefore, a trajectory more than $1 \mathrm{~mm}$ away from the structure, with sufficient irrigation, would be safe to drill. Since there is no contact of the drill with the ossicular chain, the chances of trauma are decreased. The same results were observed when analyzing the distance of the drill's trajectory to the tympanic membrane. By measuring the distance from the cortex of the temporal bone and the scala tympani, a safe, narrow, linear path via the facial recess can be drilled.

An interesting statistical difference between the position of the tympanic membrane on the left and the right sides can be seen in our tables. Valavanis, in $1983,{ }^{24}$ showed that the cranial growth process during the first 4 years of life causes changes in the position of the mastoid portion, the stylomastoid portion and the chorda tympani nerve, and the same findings were confirmed by Evangelos in 2009. ${ }^{12}$ These changes can be observed on different sides of the same specimens, demonstrating an asymmetrical growth that interferes with the position of the reference structures, such as the tympanic membrane and the chorda tympani nerve. We still need to consider the possibility that this statistical difference between the two sides occurred because of the size of our sample. Although big samples of cadavers of fetuses available for dissection are hard to find, for our objective, the number was sufficient to demonstrate the viability of the procedure.

The distances measured showed that the trajectory obtained using the CT scan did not reach any of the middle ear structures that were used as parameters, thus demonstrating that the middle ear, at this age, has enough size to allow for cochlear implant surgery. This procedure is not yet indicated because there is no sure diagnose of deafness this early in life, also because there is a need to build softer and more delicate surgical materials and guidance hardware to be used in such a fragile anatomy.

More studies are needed to better understand how the temporal bone anatomy changes during growth. Our statistical study showed an asymmetry on the position of the tympanic membrane, which also demonstrated that these changes may not be happening at the same rate on both sides of the same specimens.

By observing the measurements made, we concluded that the drill was at a safe distance from the structures, and that by as early as 32 weeks, the middle ear cavity enables safe access to the scala tympani through the facial recess.
The importance of this study resides on the fact that, in the future, in order to perform cochlear implant surgery on neonates or on infants before 5 months of life (once the difficulties of precisely diagnosing severe or profound hearing loss, at this age are overcome), it is of extreme importance to use a technique that is fast and minimally invasive, due to the limitations of the anesthetics and the possible blood loss during surgery.

\section{Conclusion}

The measurements of the relationship between the drill and the anatomical structures of the middle ear (the facial nerve, ossicular chain and tympanic membrane), and the simulation of the trajectory between the cortical temporal bone and the scala tympani, showed that none of the structures studied were damaged by the drill, which implies that, at 32 weeks, the middle ear cavity already has sufficient size to support a surgical procedure. Although cochlear implantation is not indicated at this age, this surgical technique may be a possibility in the future.

\section{Conflicts of Interest}

The authors have no conflicts of interest to declare.

\section{References}

1 Baraky LR. Prevalência de surdez incapacitante no município de Juiz de Fora, Minas Gerais, Brasil [thesis]. São Paulo: Faculdade de Medicina da Universidade de São Paulo; 2011

2 Bento RF, Martins GSQ, Pinna MH. Tratado de Otologia. 2 ed. São Paulo: Atheneu; 2013

3 Moore BCJ. Cochlear Hearing Loss: Physiological, Psychological and Technical Issues. 2 ed. West Sussex England: John Wiley \& Sons, Ltd; 2007

4 Brackmann S. Arriaga. Otologic Surgery. 3 ed. Philadelphia: Saunders; 2010

5 Bento RF. Introdução. In: Ricardo Ferreira Bento, Luiz Rodolpho Penna Lima Junior, Robinson Koji Tsuji, Maria Valéria Schmidt Goffi-Gomez, Danielle do Valle Silva Penna Lima e Rubens de Brito Neto. Tratado de implante coclear e próteses auditivas implantáveis. Rio de Janeiro. Thieme Publicações Ltda.; 2014: XIX-XXIV

6 Weiglein AH. Postnatal development of the facial canal. An investigation based on cadaver dissections and computed tomography. Surg Radiol Anat 1996;18(02):115-123

7 Lloyd SKWKA, Kasbekar AV, Kenway B, et al. Developmental changes in cochlear orientation-implications for cochlear implantation. Otol Neurotol 2010;31(06):902-907

8 Litton WBKC, Krause CJ, Anson BA, Cohen WN. The relationship of the facial canal to the annular sulcus. Laryngoscope 1969;79(09): 1584-1604

9 Migirov L, Kronenberg J. Radiology of the petromastoid canal. Otol Neurotol 2006;27(03):410-413

10 Swarts JD, Foley S, Alper CM, Doyle WJ. A cross-sectional study of the change in mastoid geometry with age in children without a history of otitis media. Laryngoscope 2012;122(03):649-653

11 Cinamon U. The growth rate and size of the mastoid air cell system and mastoid bone: a review and reference. Eur Arch Otorhinolaryngol 2009;266(06):781-786

12 Manolis EN, Filippou DK, Tsoumakas C, et al. Radiologic evaluation of the ear anatomy in pediatric cholesteatoma. J Craniofac Surg 2009;20(03):807-810 
13 Moberly AC, Lowenstein JH, Nittrouer S. Early Bimodal Stimulation Benefits Language Acquisition for Children With Cochlear Implants. Otol Neurotol 2016;37(01):24-30

14 Achiques MT, Morant A, Muñoz N, et al. Complicaciones y fallos de la implantación coclear. Acta Otorrinolaringol Esp 2010;61(06): 412-417

15 Bielamowicz SACN, Coker NJ, Jenkins HA, Igarashi M. Surgical dimensions of the facial recess in adults and children. Arch Otolaryngol Head Neck Surg 1988;114(05):534-537

16 Fayad JN, Wanna GB, Micheletto JN, Parisier SC. Facial nerve paralysis following cochlear implant surgery. Laryngoscope 2003;113(08):1344-1346

17 Warren FM, Balachandran R, Fitzpatrick JM, Labadie RF. Percutaneous cochlear access using bone-mounted, customized drill guides: demonstration of concept in vitro. Otol Neurotol 2007; 28(03):325-329

18 Labadie RFNJ, Noble JH, Dawant BM, Balachandran R, Majdani O, Fitzpatrick JM. Clinical validation of percutaneous cochlear implant surgery: initial report. Laryngoscope 2008;118(06):1031-1039
19 Wanna GB, Balachandran R, Majdani O, Mitchell J, Labadie RF. Percutaneous access to the petrous apex in vitro using customized micro-stereotactic frames based on image-guided surgical technology. Acta Otolaryngol 2009; $\cdots: 1-6$

20 Majdani O, Schurzig D, Hussong A, et al. Force measurement of insertion of cochlear implant electrode arrays in vitro: comparison of surgeon to automated insertion tool. Acta Otolaryngol 2010;130(01):31-36

21 Noble JHWF, Labadie RF, et al. Determination of drill paths for percutaneous cochlear access accounting for target positioning error. Proc SPIE 2007;6509:650-925

22 Balachandran R, Mitchell JE, Blachon G, et al. Percutaneous cochlear implant drilling via customized frames: an in vitro study. Otolaryngol Head Neck Surg 2010;142(03):421-426

23 Cohen NL. Cochlear implant soft surgery: fact or fantasy? Otolaryngol Head Neck Surg 1997;117(3 Pt 1):214-216

24 Valavanis A, Kubik S, Oguz M. Exploration of the facial nerve canal by high-resolution computed tomography: anatomy and pathology. Neuroradiology 1983;24(03):139-147 